\title{
Impact of an intervention programme on knowledge, attitudes and practices of population regarding severe fever with thrombocytopenia syndrome in endemic areas of Lu'an, China
}

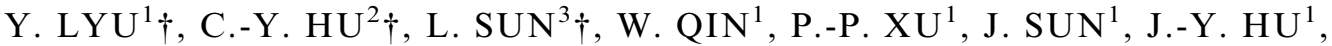 \\ Y. YANG ${ }^{1}$, F.-L. LI ${ }^{2}$, H.-W. CHANG ${ }^{1}$, X.-D. LI ${ }^{1}$, S.-Y. XIE ${ }^{1}$, K.-C. LI ${ }^{1}$, \\ X.-X. HUANG ${ }^{4}$, F. DING ${ }^{4}$ AND X.-J. ZHANG ${ }^{2}$ \\ ${ }^{1}$ Lu'an City Center for Disease Control and Prevention, Lu'an, China \\ ${ }^{2}$ Department of Epidemiology and Biostatistics, School of Public Health, Anhui Medical University, Hefei, China \\ ${ }^{3}$ Fuyang City Center for Disease Control and Prevention, Fuyang, China \\ ${ }^{4}$ Chinese Center for Disease Control and Prevention, Beijing, China
}

Received 3 July 2017; Final revision 25 October 2017; Accepted 2 November 2017; first published online 27 November 2017

\section{SUMMARY}

Knowledge, attitudes and practices (KAP) of the population regarding severe fever with thrombocytopenia syndrome (SFTS) in endemic areas of Lu'an in China were assessed before and after an intervention programme. The pre-intervention phase was conducted using a sample of 425 participants from the 12 selected villages with the highest rates of endemic SFTS infection. A predesigned interview questionnaire was used to assess KAP. Subsequently, an intervention programme was designed and applied in the selected villages. KAP was re-assessed for each population in the selected villages using the same interview questionnaire. Following 2 months of the programme, 339 participants had completed the re-assessed survey. The impact of the intervention programme was evaluated using suitable statistical methods. A significant increase in the KAP and total KAP scores was noted following the intervention programme, whereas the proportion of correct knowledge, the positive attitudes and the effective practices toward SFTS of respondents increased significantly. The intervention programme was effective in improving KAP level of SFTS in populations that were resident in endemic areas.

Key words: Impact, intervention programme, KAP, severe fever with thrombocytopenia syndrome.

\section{INTRODUCTION}

Severe fever with thrombocytopenia syndrome (SFTS) is an emerging tick-borne infectious disease characterised by fever, thrombocytopenia and leukocytopenia. The disease was caused by the SFTS virus (SFTSV), a newly identified member of the genus

\footnotetext{
* Author for correspondence: X.-J. Zhang, Department of Epidemiology and Biostatistics, School of Public Health, Anhui Medical University, 81 Meishan Road, Hefei 230032, Anhui, People's Republic of China.

(Email: zxj731024@126.com)

$\uparrow$ These authors contributed equally to this work.
}

Phlebovirus, family Bunyavirudae [1, 2]. SFTS has been reported in China [3], South Korea [4] and Japan [5], with an average mortality rate of $5 \cdot 3 \%$ in China [6]. From 2010 to 2016, SFTS had been reported in 23 provinces of China, with increased numbers of people infected with SFTSV yearly. The infection and death cases were mainly found in central China, where the Haemaphysalis longicornis ticks are spread [6].

Previous studies have demonstrated that people who work outdoors and/or live in mountainous or hilly rural areas were considered as a high-risk population and preventative measures that reduce exposure 
to ticks can prevent SFTSV infection [7-9]. Owing to the unavailability of vaccines against SFTS and the fatal outcomes of the syndrome, considerable attention should be paid to promoting and strengthening health education and behavioral change among highrisk populations, especially for people who work outdoors in the SFTS endemic areas. The health education of the affected population can promote its awareness and preventive skills against SFTS [10]. In addition, it can strengthen the multi-sectoral joint cooperation mechanism and increase the social health benefits that are provided by the government with regard to SFTSV infection.

A 'Knowledge, Attitudes, and Practices' (KAP) survey is representative of a specific population and is used in order to collect information on what is known, believed and done regarding a specific topic [11]. It is required for program implementers to understand the disease-related KAP of the affected population since KAP is an important determinant of community involvement and collaboration and plays a crucial role in the successful prevention and control of the disease [12]. Certain studies have shown that the improved KAP level of a population can effectively prevent and control vector-borne diseases such as Rift Valley fever, malaria and dengue fever [13-15]. The public's knowledge of SFTS, the attitudes to risks and adaptation practices comprise three of the most important factors for the reduction of the risk of SFTS infection. It is therefore recommended that the population in endemic areas should be provided with targeted health education regarding proper preventive and control strategies for SFTS.

Although numerous studies have been conducted for the assessment of levels of different disease-related KAP in different regions of the world [16-20], to our knowledge, no SFTS-related KAP studies have been reported. Therefore, the present study was conducted among populations in endemic areas of Lu'an city. The city is known as the western region of Anhui province and is located in the middle-eastern region of China, accounting for $20.76 \%$ of the total number of confirmed disease cases in the Anhui province from January 2011 to July 2014 [21]. The ultimate goal of the study was to provide evidence to the local health authorities and communities for the development of preventive strategies that aim to reduce the incidence of SFTS. The present study provides baseline data with regard to SFTS-related KAP of a local population in China. The data can be used for future investigations regarding SFTS prevention and control. The study findings further provide baseline data that could be used for the evaluation of the intervention effects on the KAP survey items.

\section{METHODS}

The present pre-post intervention study was conducted from September 2016 to April 2017. The study consisted of three phases: pre-intervention phase; intervention phase; and post-intervention phase.

\section{Pre-intervention phase}

The pre-intervention phase was conducted in SFTS endemic areas of Lu'an city. In order to represent the areas of endemic infection, we first selected two counties with the highest rates of endemic SFTS infection from 2011 to 2016 according to the China Information System for Diseases Control and Prevention (CISDCP); Second, three towns with the highest rates of endemic SFTS infection of the selected counties were included in the study; Finally, we selected two villages with the highest rates of endemic SFTS infection from the selected towns. Therefore, a total of 12 villages were selected from the six towns in two counties (Jinzhai county and Huoshan county) of Lu'an city. The 12 selected villages were located at the northern foot of the Dabie Mountains with forest coverage rate of $75 \%$. The climate feature of these areas is warm and humid, with an annual average temperature of $15.6{ }^{\circ} \mathrm{C}$. These natural conditions are favourable for the growth and reproduction of ticks. A previous study that was conducted in these areas used targeted surveillance and then indicated that the two counties were endemic areas [21]. The study populations consisted of two groups (students of fourth, fifth and sixth grades in primary schools and ordinary residents) living and/or working in the selected villages at least 3 months. Primary school students were further included as study populations for the following two reasons: First, the students in the rural primary schools in the areas investigated in the present study were mostly left-behind children. Their parents migrated from underdeveloped rural areas to urban centers in search of optimal job opportunities. The majority of the children were resident in hometowns with their grandparents. The children were involved in farm work activities including tea-picking and feeding livestock. They were also vulnerable to exposure to ticks. Second, the aim of the study was the education of selected children so that they could 
spread the knowledge acquired from the intervention programme to their guardians since the majority of their guardians had no formal education. Healthcare staff including doctors and nurses was not included as their special occupations. The calculation of the sample size and sampling diagram for the present study is presented in Eq. (1) [14] and Fig. 1, respectively.

$n=\frac{Z^{2} p(1-p)}{d^{2}}=\frac{(1 \cdot 96)^{2}(0 \cdot 50)(1-0 \cdot 50)}{(0 \cdot 05)^{2}}=384$

where $n=$ the sample size; $d=$ an acceptance error $=$ $0.1 \times p ; Z=1.96$ (the standard normal score at the $95 \%$ confidence interval); $P=0 \cdot 50$ (assume the proportion of participants knowledgeable on SFTS is 50\%).

The sample size was increased by $10 \%$ to account for unresponsive subjects, missing and/or incomplete data that occurred during data collection. Consequently, the required sample size was at least 422 individuals.

A structured questionnaire was used to collect the demographic information and assess the KAP of the population with regard to SFTS. The questionnaire was developed with reference to expert consultation and the 'Guidelines for the prevention and control of severe fever with thrombocytopenia syndrome (2010 edition)' published by the Chinese ministry of health in order to match the scope of the study (http://www. moh.gov.cn/mohwsyjbgs/s8348/201010/49272.shtml). The questionnaire consisted of four major sections: the first section was concerned with the demographic data of participants and the second; third; and fourth sections were formulated in order to test the knowledge; attitudes; and practices of the subjects toward SFTS. A total of six questions were used in order to assess the knowledge, while the practices were assessed using three questions. The attitudes were evaluated by five statements that were presented on a three-point Likert scale format with 'Yes', 'maybe', and 'no' responses. For each question in the knowledge section, a score of 1 was awarded for each correct response while a wrong response or 'don't know' corresponded to a score of 0 . A knowledge score for each respondent was calculated by summing the number of correct answers collected out of six questions. A score of 2 was awarded if the respondent reported 'Yes', while 'maybe' and 'no' responses were assigned to 1 and 0 scores in the attitudes section, respectively. Each correct preventive measure of respondents mentioned was awarded a score of 1 , while a score of 0 was assigned if the preventive measure was incorrect and/ or not mentioned. A practice score for each respondent was calculated by summing the number of correct responses out of the three questions. Thus, the maximum total score for the questionnaire was 26 .

Prior to data collection, the validity of the designed questionnaire was confirmed by a Cronbach's alpha $(\alpha)$ internal consistency coefficient of 0.83 for the three components in a pilot study involving 30 participants with different occupations (farmers, students, workers and self-employed works, etc.). Following the pilot study and through research team discussion, the decision was made to use the questionnaire as an interview questionnaire rather than a selfadministered questionnaire, since certain items required explanation to the participants by trained interviewers.

Various measures were taken in order to ensure the quality of the questionnaire. All interviewers received systematic training including roles, responsibilities and appropriate interviewing skills prior to the survey. Furthermore, during questionnaire collection, two senior researchers were always present to monitor the process and examine the quality of the data collected.

\section{Intervention phase}

The Information-Motivation-Behavioral Skills (IMB) model is a theoretical framework developed by Fisher et al. [22, 23]. The IMB model of health behaviour modification using the assumption that executing a health promotion behaviour is a function of the extent to which one is well informed regarding the behaviour and/or motivated to engage in the behaviour. For example, the model can assess whether one participant has positive personal attitudes and/or intentions toward the behaviour and social support for the behaviour. Furthermore, the IMB model assesses whether the subjects have the appropriate behavioural skills to perform the behaviour [24]. Briefly, this model postulates that prevention behaviours are directly influenced by an individual's knowledge regarding a disease, their motivation to avoid the disease and their skills and capacity to engage in behaviours, while the information and motivation further cause a direct influence in the behavioural skills. Based on the assumption and the study results in the pre-intervention phase, an educational programme was designed and carried out in the 12 villages. This programme was tailor-made according to the specific needs of the rural residents and primary school students. The educational programme was offered by way of integrating 


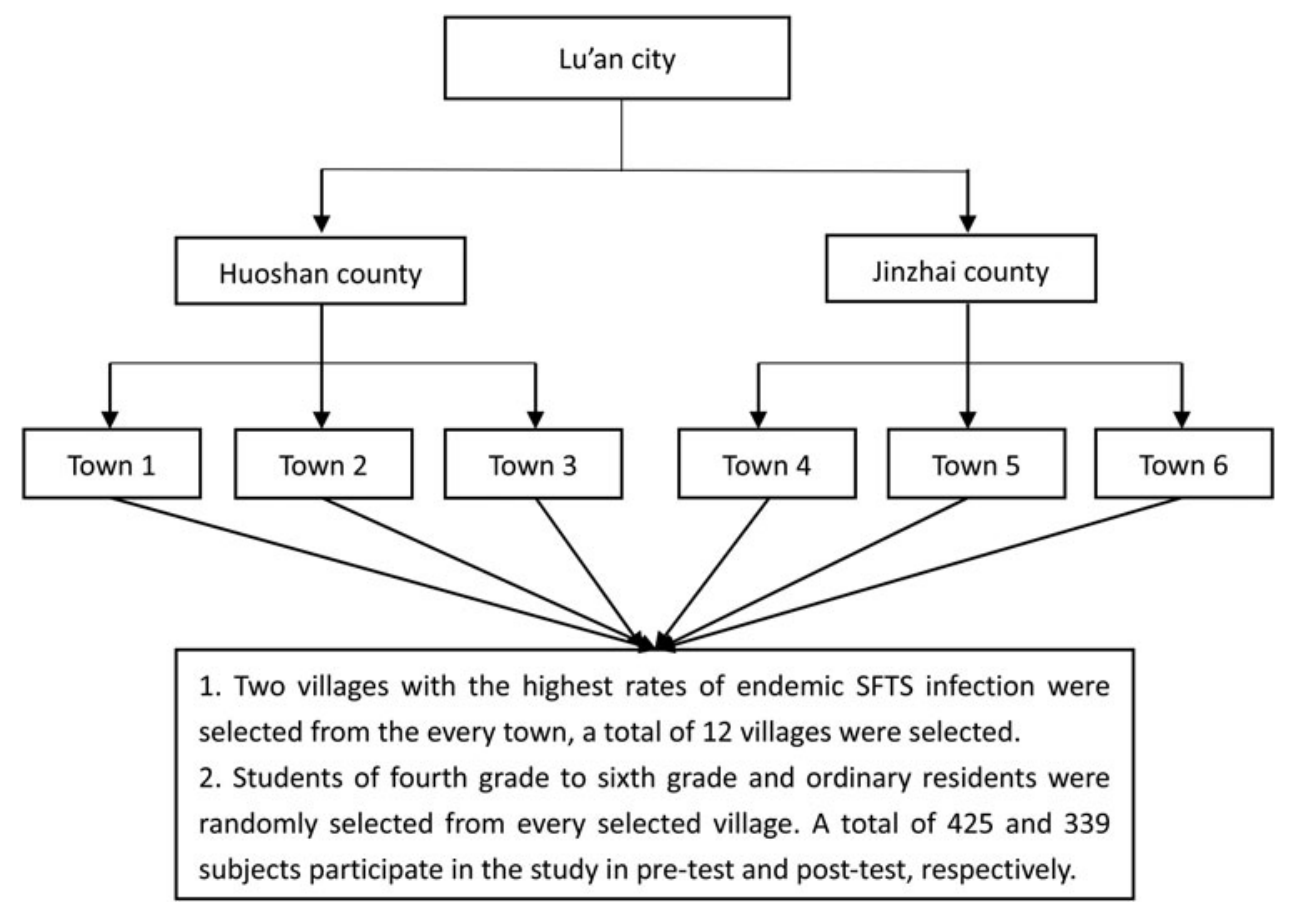

Fig. 1. Sampling diagram of the study.

community-level and individual-level in the 12 villages, which were considered as 12 community units. The specific measures were as follows: (1) the basic preventive knowledge of SFTS was spread to the community population by personalised health communication materials including posters, banners and propaganda brochures, which were developed based on expert consultation; (2) SFTS epidemic was most common in rural areas, due to the relatively low educational level of the rural residents and due to difficult access to relevant information. Therefore, the rural primary school students were responsible to spread the preventive knowledge to their families based on the acquired knowledge from the health education lectures and the peer discussions, which were conducted in the community primary schools; (3) the combined measures of the community health education and individual counselling were used due to the existence of individual differences. The local clinicians further provided individual health education counseling and interaction with subjects who exhibited lower awareness of SFTS in the pre-intervention phase; (4) during the intervention period, health education activities were facilitated via the use of appropriate mass media including radio, television, newspapers and internet. Finally, the village clinicians required specific training in order to improve their diagnostic and treatment ability with regard to SFTS.

\section{Post-intervention phase}

The intervention programme was conducted specifically between late January and early February. Following 2 months of conduct, the second survey was carried out in the post-intervention phase in order to reassess the KAP of the population regarding SFTS. This was achieved by the same interview questionnaire and scoring criteria for each question in the pre-intervention phase. Since most rural populations in these areas had initiated their farming activities, including tea-picking, in April, they were more vulnerable to tick exposure. Consequently, the second survey was conducted in order to reassess the KAP and improve the awareness of the populations in these areas. According to the sampling procedure in the preintervention phase, we calculated the sample size with the knowledge rate of $56.2 \%$, based on the results obtained in the first survey and increased by $10 \%$ in order to allow any missing and/or incomplete data in the post-intervention phase. Consequently, the required sample size was at least 332 . Finally, a total of 339 participants completed the re-assessed survey.

\section{Data analysis}

The data were coded and entered into EpiData 3.1 and the collected data were analysed using SPSS 
version 11. Assessment of the normality of data was performed using Shapiro-Wilk test. Significant results for total KAP, KAP scores $(P \mathrm{~s}<0 \cdot 05)$ were obtained. For categorical variables, the data were computed as frequencies and percentages, while for continuous variable there was presented as mean \pm standard deviation (s.D.), such as the parameters of age. For non-normally distributed data including total KAP, KAP scores were described as median and interquartile range (IQR). The comparison of the proportions for the categorical variables was carried out using the $\chi^{2}$ test or Fisher's exact test when appropriate. The total KAP, KAP scores in the pre-test and the post-test were compared using the Wilcoxon Rank Tests in order to determine the impacts of the intervention programme. Tests were two-tailed. A $P$ value of less than $0.05(P<$ $0 \cdot 05)$ was considered statistically significant.

\section{Ethical statement and informed consent}

Ethical approval was obtained from the Biomedical Ethics Committee of the Anhui Medical University (No. 2015005). Written informed consent was obtained from all the participants prior to the survey. All data obtained were anonymous and all attendees were offered the option of not participating.

\section{RESULTS}

\section{Demographic characteristics of the participants}

The parameters of age, sex, educational level, occupation and frequency of outdoor work during the last month of the participants in the pre- and postintervention phases are presented in Table 1. The mean age of the respondents was 39.90 (s.D., 22.12) years in the pre-intervention phase and 38.82 (s.D., 21.98) years in the post-intervention phase. Among the 425 respondents in the pre-intervention phase, $51 \cdot 8 \%$ were male and $49 \cdot 2 \%$ had an elementary school educational level. Similarly, $49 \cdot 6 \%$ among the 339 respondents in the post-intervention phase were male and $44.5 \%$ had an elementary school educational level. In terms of occupation, the majority of the respondents were farmers and students in the two phases. With regard to the frequency of the outdoor work during the last month, $36.7 \%$ of the respondents worked outdoors occasionally (1-9 days last month) and $29 \cdot 6 \%$ of these respondents usually worked outdoors during the last month ( $\geqslant 20$ days last month) in the pre-intervention phase. In the post-intervention phase, $38 \cdot 3 \%$ of respondents worked outdoors occasionally and $25 \cdot 7 \%$ usually worked outdoors during the last month. No significant differences were observed between the two study phases in terms of age, sex, educational level, occupation and frequency of outdoor work during the last month $(P>0 \cdot 05)$.

\section{Impact of the intervention programme on KAP of students and non-students groups}

The KAP scores of the students group and the nonstudents group before and after the intervention programme were statistically tested by Wilcoxon rank test at $95 \%$ confidence interval to evaluate the difference between the two groups. In the pre-intervention phase, the total KAP scores for students group (mean rank $=166 \cdot 6$ ) and non-students group (mean rank $=235 \cdot 1$ ) were statistically significantly different and displayed a median of $12(\mathrm{IQR}=9 \cdot 5-14)$ in students group and 14 (IQR $=11-17)$ in non-students group, respectively. In the post-intervention phase, the total KAP scores for students group (mean rank $=176 \cdot 4$ ) and non-students group (mean rank $=167 \cdot 1$ ) were not statistically significantly different and displayed a median of 17 (IQR = 15-19) in students group and 17 (IQR = 13-19) in nonstudents group, respectively. Based on these results, it can be assumed that the intervention programme had a better effect on students group.

\section{Impact of the intervention programme on knowledge}

We assessed KAP of respondents regarding SFTS based on their responses to the questionnaire provided. Table 2 shows the median (IQR) of KAP and total KAP score was $3(1-5), 8(6 \cdot 5-9), 3(2-3)$ and $13(11-17)$ in the pre-invention phase and $5(3-7)$, 10 (7-10), 3 (3-3) and 17 (14-19) in the post-invention phase, respectively. The results indicated that there was a significant increase in the four type scores $(P s<$ $0 \cdot 001$ ). As shown in Table $3,56 \cdot 2 \%$ out of the 425 respondents in the pre-intervention phase reported initially that they had knowledge of SFTS, whereas following the educational programme, this percentage was increased to $80 \cdot 4 \%$ out of 339 respondents. This indicated a mean increase of $24.2 \% \quad(P<0.001)$. Approximately half of the respondents $(53.6 \%)$ reported that fever was the most common symptom of SFTS and other symptoms such as general malaise, fatigue, headache, muscle pain; nausea, vomit, anorexia, diarrhea; thrombocytopenia; leukopenia; and cough, sore throat were less frequently reported as the most common symptom as cited by $32.0 \%$, 
Table 1. Sample socio-demographic characteristics from the two study phases (pre-intervention phase and post-intervention phase)

\begin{tabular}{|c|c|c|c|}
\hline Variables & $\begin{array}{l}\text { Pre-intervention phase } \\
(n=425) \\
n(\%)\end{array}$ & $\begin{array}{l}\text { Post-intervention phase } \\
(n=339) \\
n(\%)\end{array}$ & $P$ value \\
\hline Age (years) & & & $0 \cdot 270$ \\
\hline Less than 25 & $128(30 \cdot 0)$ & $102(30 \cdot 1)$ & \\
\hline $25-50$ & $130(30 \cdot 5)$ & $87(25 \cdot 7)$ & \\
\hline 51 and above & $168(39 \cdot 4)$ & $150(44 \cdot 2)$ & \\
\hline Sex & & & $0 \cdot 545$ \\
\hline Male & $220(51 \cdot 8)$ & $167(49 \cdot 6)$ & \\
\hline Female & $205(48 \cdot 2)$ & $170(50 \cdot 4)$ & \\
\hline Educational level & & & $0 \cdot 365$ \\
\hline Illiteracy & $64(15 \cdot 1)$ & $46(14 \cdot 4)$ & \\
\hline Elementary school & $209(49 \cdot 2)$ & $142(44 \cdot 5)$ & \\
\hline Junior middle school & $67(15 \cdot 8)$ & $64(20 \cdot 1)$ & \\
\hline Senior middle school/Secondary vocational school & $61(14 \cdot 4)$ & $42(13 \cdot 2)$ & \\
\hline Three-year university degree or above & $24(5 \cdot 6)$ & $25(7 \cdot 8)$ & \\
\hline Occupation & & & $0 \cdot 810$ \\
\hline Student & $142(33 \cdot 4)$ & $109(32 \cdot 2)$ & \\
\hline Unemployed & $30(7 \cdot 1)$ & $23(6 \cdot 8)$ & \\
\hline Migrant worker & $8(1 \cdot 9)$ & $4(1 \cdot 2)$ & \\
\hline Farmer & $153(36 \cdot 0)$ & $122(36 \cdot 0)$ & \\
\hline Teacher & $21(4 \cdot 9)$ & $22(6 \cdot 5)$ & \\
\hline Self-employed individual & $32(7 \cdot 5)$ & $34(10 \cdot 0)$ & \\
\hline Township cadre & $28(6 \cdot 6)$ & $19(5 \cdot 6)$ & \\
\hline Others & $11(2 \cdot 6)$ & $6(1 \cdot 8)$ & \\
\hline Frequency of outdoor work during the last month & & & $0 \cdot 098$ \\
\hline Usually ( $\geqslant 20$ days last month) & $126(29 \cdot 6)$ & $87(25 \cdot 7)$ & \\
\hline Often (10-19 days last month) & $79(18 \cdot 6)$ & $51(15 \cdot 0)$ & \\
\hline Occasionally (1-9 days last month) & $156(36 \cdot 7)$ & $130(38 \cdot 3)$ & \\
\hline Never & $64(15 \cdot 1)$ & $71(20 \cdot 9)$ & \\
\hline
\end{tabular}

$P$-value; $\chi^{2} /$ Fisher Exact Test estimation.

Table 2. Differences in knowledge, attitude, practice and total (overall) (shown as median (interquartile range)) scores in pre-intervention phase and post-intervention phase

\begin{tabular}{llllll}
\hline \hline Domain & Pre-test score & Pre-test mean rank & Post-test score & Post-test mean rank & $P$ value \\
\hline Knowledge & $3(1-5)$ & $332 \cdot 1$ & $5(3-7)$ & $445 \cdot 6$ & $<0 \cdot 001$ \\
Attitude & $8(6 \cdot 5-9)$ & $326 \cdot 1$ & $10(7-10)$ & $453 \cdot 2$ & $<0 \cdot 001$ \\
Practice & $3(2-3)$ & $328 \cdot 0$ & $3(3-3)$ & $450 \cdot 9$ & $<0 \cdot 001$ \\
Overall & $13(11-17)$ & $322 \cdot 2$ & $17(14-19)$ & $458 \cdot 1$ & $<0 \cdot 001$ \\
\hline \hline
\end{tabular}

$P$ value; Wilcoxon Rank Tests.

$16 \cdot 9 \%, 16 \cdot 7 \%, 8 \cdot 7 \%$ and $6 \cdot 6 \%$ of the respondents, respectively. Following the intervention programme, the proportions of participants that reported symptoms such as nausea, vomit, anorexia, diarrhoea $(30 \cdot 4 \%)$; thrombocytopenia $(29 \cdot 5 \%)$; leukopenia $(23 \%)$; and cough, sore throat $(10 \cdot 6 \%)$ were significantly increased. A significantly increased proportion of participants reported that most SFTS cases can be cured $(50 \cdot 7 \%$ vs. $64 \cdot 4 \% ; P<0 \cdot 001)$, the proportion of participants who believed that SFTS cannot be prevented with the vaccine was significantly increased following the intervention programme (16.9\% vs. $24 \cdot 6 \%$; $P<0.001)$. A similar result was noted for the proportion of participants who believed that SFTS can be infectious to others $(28.6 \%$ vs. $41.8 \% ; P<0.001)$ following the intervention programme. A substantial number of respondents in the two phases believed that if they live with the patient, contact or speak 
Table 3. Differences in knowledge in pre-intervention phase and post-intervention phase

\begin{tabular}{|c|c|c|c|}
\hline \multirow[b]{2}{*}{ Knowledge questions } & \multicolumn{2}{|l|}{ Test responses $(\%)$} & \multirow[b]{2}{*}{$P$ value } \\
\hline & $\begin{array}{l}\text { Pre-intervention Phase } \\
(n=425)\end{array}$ & $\begin{array}{l}\text { Post-intervention Phase } \\
(n=339)\end{array}$ & \\
\hline Do you know what SFTS is? & & & $<0.001$ \\
\hline Yes & $237(56 \cdot 2)$ & $271(80 \cdot 4)$ & \\
\hline No & $185(43 \cdot 8)$ & $66(19 \cdot 6)$ & \\
\hline \multicolumn{4}{|l|}{ What are the most common symptoms of SFTS? } \\
\hline Fever* & $228(53 \cdot 6)$ & $203(59 \cdot 6)$ & $0 \cdot 084$ \\
\hline General malaise, fatigue, headache, muscle pain* & $136(32 \cdot 0)$ & $126(37 \cdot 2)$ & $0 \cdot 135$ \\
\hline Nausea, vomit, anorexia, diarrhoea* & $72(16 \cdot 9)$ & $103(30 \cdot 4)$ & $<0 \cdot 001$ \\
\hline Cough, sore throat* & $28(6 \cdot 6)$ & $36(10 \cdot 6)$ & $0 \cdot 046$ \\
\hline Leukopenia* & $37(8 \cdot 7)$ & $78(23 \cdot 0)$ & $<0.001$ \\
\hline Thrombocytopenia* & $71(16 \cdot 7)$ & $100(29 \cdot 5)$ & $<0.001$ \\
\hline Do you think that SFTS can be cured? & & & $0 \cdot 001$ \\
\hline All cases can be cured & $32(7 \cdot 6)$ & $18(5 \cdot 4)$ & \\
\hline Most cases can be cured after regular treatment* & $214(50 \cdot 7)$ & $215(64 \cdot 4)$ & \\
\hline Don't know & $176(41 \cdot 7)$ & $101(30 \cdot 2)$ & \\
\hline Do you think that SFTS can be prevented with a vaccine? & $81(19 \cdot 2)$ & $90(26 \cdot 7)$ & $<0 \cdot 001$ \\
\hline Yes & $71(16 \cdot 9)$ & $83(24 \cdot 6)$ & \\
\hline No* & $71(16 \cdot 9)$ & $83(24 \cdot 6)$ & \\
\hline Don't know & $269(63 \cdot 9)$ & $164(48 \cdot 7)$ & \\
\hline Do you think that SFTS can be infectious to others? & & & $<0 \cdot 001$ \\
\hline Yes* & $118(28 \cdot 6)$ & $141(41 \cdot 8)$ & \\
\hline No & $120(29 \cdot 1)$ & $107(31 \cdot 8)$ & \\
\hline Don't know & $175(42 \cdot 4)$ & $89(26 \cdot 4)$ & \\
\hline \multicolumn{4}{|c|}{$\begin{array}{l}\text { Which of the following human activities may cause spreading of the } \\
\text { disease? }\end{array}$} \\
\hline Living with the patient & $52(12 \cdot 2)$ & $50(14 \cdot 7)$ & $0 \cdot 310$ \\
\hline Contacting or speaking with the patient closely & $42(9 \cdot 9)$ & $51(15 \cdot 0)$ & $0 \cdot 030$ \\
\hline Contacting with the patient's blood and/or secretions directly* & $108(25 \cdot 4)$ & $144(42 \cdot 5)$ & $<0 \cdot 001$ \\
\hline Tick bite* & $187(44)$ & $130(38 \cdot 3)$ & $0 \cdot 115$ \\
\hline
\end{tabular}

SFTS, severe fever with thrombocytopenia syndrome.

$P$-value; $\chi^{2} /$ Fisher Exact Test estimation.

* Correct items.

with the patient closely can cause the spreading of SFTS. Only $25 \cdot 4 \%$ of the respondents were aware that contact with the blood and/or secretions of SFTS patients directly can cause the spreading of SFTS. This proportion increased to $42 \cdot 5 \%$ following the intervention programme $(P<0 \cdot 001)$. However, a decreased proportion of respondents were noted who believed that tick bite can cause the spreading of SFTS, although this did not reach statistical significance.

\section{Impact of the intervention programme on attitudes}

Table 4 indicates the respondents' attitudes toward the prevention of SFTS with regard to adopting personal protective strategies when in contact with livestock; dealing with livestock that carries ticks in time to prevent family members from being bitten by ticks; cleaning up weeds and shrubs in the yard and/or around the house regularly; and participating in the free related detections of SFTS that were conducted by health departments. The proportion of the participants significantly increased from 56.9\%, $73 \cdot 7 \%, 80 \cdot 0 \%$ and $66 \cdot 9 \%$ to $68 \cdot 2 \%, 79 \cdot 6 \%, 84 \cdot 8 \%$ and $81.0 \%$, respectively, following the intervention programme, with exception of the attitudes about counselling-related information about preventive strategies of SFTS in the lectures or propaganda which were conducted in communities.

\section{Impact of the intervention programme on practices}

With regard to practice questions (Table 5 ), $68.0 \%$ of the respondents believed that if someone who was bitten by tick, he or she should palsy tick with medical 
Table 4. Differences in attitudes in pre-intervention phase and post-intervention phase

\begin{tabular}{|c|c|c|c|}
\hline \multirow[b]{2}{*}{ Attitude questions } & \multicolumn{2}{|l|}{ Test responses $(\%)$} & \multirow[b]{2}{*}{$P$ value } \\
\hline & Pre-intervention Phase $(n=425)$ & Post-intervention Phase $(n=339)$ & \\
\hline \multicolumn{3}{|c|}{$\begin{array}{l}\text { Would you adopt personal protective strategies when in contact with livestock (e.g. sheep, cattle, pigs, } \\
\text { dogs, etc.)? }\end{array}$} & $0 \cdot 006$ \\
\hline Yes & $236(56 \cdot 9)$ & $227(68 \cdot 2)$ & \\
\hline No & $116(28 \cdot 0)$ & $66(19 \cdot 8)$ & \\
\hline Maybe & $63(15 \cdot 1)$ & $40(12 \cdot 0)$ & \\
\hline \multicolumn{3}{|c|}{$\begin{array}{l}\text { Would you deal with livestock that carries ticks in time to prevent family members from being bitten by } \\
\text { ticks? }\end{array}$} & $0 \cdot 006$ \\
\hline Yes & $302(73 \cdot 7)$ & $265(79 \cdot 6)$ & \\
\hline No & $78(19 \cdot 0)$ & $36(10 \cdot 8)$ & \\
\hline Maybe & $30(7 \cdot 3)$ & $32(9 \cdot 6)$ & \\
\hline \multicolumn{3}{|c|}{ Would you clean up weeds and shrubs in the yard and/or around the house regularly? } & $0 \cdot 036$ \\
\hline Yes & $328(80 \cdot 0)$ & $285(84 \cdot 8)$ & \\
\hline No & $63(15 \cdot 4)$ & $31(9 \cdot 2)$ & \\
\hline Maybe & $19(4 \cdot 6)$ & $20(6 \cdot 0)$ & \\
\hline \multicolumn{3}{|c|}{$\begin{array}{l}\text { Would you counsel related information about preventive strategies of SFTS in the lectures or propaganda } \\
\text { which were conducted in your communities? }\end{array}$} & $0 \cdot 106$ \\
\hline Yes & $357(91 \cdot 9)$ & $322(95 \cdot 3)$ & \\
\hline No & $16(3 \cdot 8)$ & $7(2 \cdot 1)$ & \\
\hline Maybe & $18(4 \cdot 3)$ & $9(2 \cdot 7)$ & \\
\hline \multicolumn{3}{|c|}{ Would you participate in the free related detections of SFTS that conducted by health departments? } & $<0 \cdot 001$ \\
\hline Yes & $281(66 \cdot 9)$ & $273(81 \cdot 0)$ & \\
\hline No & $12(2 \cdot 9)$ & $4(1 \cdot 2)$ & \\
\hline Maybe & $127(30 \cdot 2)$ & $60(17 \cdot 8)$ & \\
\hline
\end{tabular}

SFTS, severe fever with thrombocytopenia syndrome. $P$-value; $\chi^{2} /$ Fisher Exact Test estimation.

alcohol or iodine and then remove the tick slowly; $90.5 \%$ of the respondents believed that if someone who was bitten by tick and developed symptoms of fever or other physical discomfort, he or she should raise vigilance and go to the hospital for treatment immediately. The proportion of the two practices significantly increased to $79 \cdot 4 \%$ and $95 \cdot 2 \%$ following the intervention programme, respectively. With regard to the use of general precautions when working outdoors, the most common practice adopted by $56 \cdot 2 \%$ of the respondents was wearing long-sleeved clothing and putting cuffs into the socks or shoes, followed by $31 \cdot 1 \%$ of the respondents applying repellent. The proportion significantly increased to $69 \cdot 9 \%$ and $46 \cdot 3 \%$ of the respondents using these two precautions, respectively following the intervention programme.

\section{DISCUSSION}

To the best of our knowledge, this is the first study that provides evidence of the impact of an intervention programme on KAP concerning SFTS conducted in rural populations randomly selected from the 12 villages in the two counties of Lu'an city. These locations are known as endemic areas of SFTS. The results could be used as baseline data in order to evaluate future tools and strategies formulated to prevent, control and/or eradicate SFTS in the study areas and other similar areas.

The compare the difference of KAP from students with that from other people group indicated that the intervention programme had a better effect on students group. So, we think that they could spread the knowledge acquired from the intervention programme to their guardians. The limited knowledge of the participants regarding SFTS was shown in the study in pre-intervention phase. The reason for this outcome may be attributed to the relatively high frequency of illiteracy and elementary school educational level among participants $(64 \cdot 3 \%)$ that play an important role in limiting the awareness and/or acquisition of knowledge regarding SFTS. Several earlier studies demonstrated that the educational level was an important determinant of knowledge acquisition 
Table 5. Differences in practices in pre-intervention phase and post-intervention phase

\begin{tabular}{|c|c|c|c|}
\hline \multirow[b]{2}{*}{ Practices questions } & \multicolumn{2}{|l|}{ Test responses $(\%)$} & \multirow[b]{2}{*}{$P$ value } \\
\hline & $\begin{array}{l}\text { Pre-intervention Phase } \\
(n=425)\end{array}$ & $\begin{array}{l}\text { Post-intervention Phase } \\
(n=339)\end{array}$ & \\
\hline \multicolumn{3}{|l|}{ What do you think someone should do if she or he was bitten by tick? } & $0 \cdot 002$ \\
\hline $\begin{array}{l}\text { Palsying tick with medical alcohol or iodine and then remove the } \\
\text { tick slowly }\end{array}$ & $272(68 \cdot 0)$ & $262(79 \cdot 4)$ & \\
\hline Squashing the tick and then removed it directly & $103(25 \cdot 8)$ & $57(17 \cdot 3)$ & \\
\hline Don't deal with it & $25(6 \cdot 2)$ & $11(3 \cdot 3)$ & \\
\hline \multicolumn{3}{|c|}{$\begin{array}{l}\text { What advice would you give someone who was bitten by tick and developed symptoms of fever or other physical } \\
\text { discomfort? }\end{array}$} & $0 \cdot 011$ \\
\hline $\begin{array}{l}\text { Raising vigilance and go to the hospital for treatment } \\
\text { immediately }\end{array}$ & $363(90 \cdot 5)$ & $316(95 \cdot 2)$ & \\
\hline Symptomatic treatment with drugs which were bought by oneself & $23(5 \cdot 7)$ & $5(1 \cdot 5)$ & \\
\hline Don't take any measures & $15(3 \cdot 7)$ & $11(3 \cdot 3)$ & \\
\hline \multicolumn{4}{|l|}{$\begin{array}{l}\text { What are the general precautions to take when you working } \\
\text { outdoors? }\end{array}$} \\
\hline Don't take protective measures & $86(20 \cdot 2)$ & $52(15 \cdot 3)$ & $0 \cdot 081$ \\
\hline Wearing long-sleeved clothes and put cuffs into the socks or shoes & $239(56 \cdot 2)$ & $237(69 \cdot 9)$ & $<0 \cdot 001$ \\
\hline Applying repellent & $132(31 \cdot 1)$ & $157(46 \cdot 3)$ & $<0 \cdot 001$ \\
\hline Don't know how to take protective measures & $50(11 \cdot 8)$ & $26(7 \cdot 7)$ & $0 \cdot 060$ \\
\hline
\end{tabular}

$P$-value; $\chi^{2} /$ Fisher Exact Test estimation.

with regard to similar vector-borne diseases $[25,26]$ and individuals with higher educational level were more likely to have sufficient knowledge compared with those who were less educated [27]. A significantly improved proportion of respondents were knowledgeable of SFTS and the most common symptoms of this disease in the post-intervention phase were noted due to the effect of intervention. Since it is crucial for a person to recognise the disease and its symptoms, and seek adequate medical help in a timely manner, this finding confirms the necessity for health education. However, the correct answer proportions of the most common symptoms was low at the initiation of the programme and following its completion, which may be explained by the fact that it is even difficult for medical workers to report the majority of clinical symptoms of SFTS in the absence of previous encounters, not to mention rural residents. Appropriately half $(50.7 \%)$ of the respondents in the pre-intervention phase believed that most cases can be cured after regular treatment. The average mortality rate of SFTS is currently estimated to $5.3 \%$ in China [6] and in some severe cases, fatalities occur due to untimely treatment. The proportions of the respondents reported that SFTS cannot be prevented with vaccines and that SFTS can be infectious to others by contacting with the patient's blood and/or secretions directly, or tick bite was relatively low in the two phases. It can be speculated that there is limited accessible source of information with regard to a previously unfamiliar disease with the exception of the intervention programme. Consequently, certain populations cannot provide sufficient information to the disease. Thus, the intervention programme should be continuous and further developed and implemented in order to educate and increase the public knowledge level of this disease.

Remarkable percentage of respondents in the two phases exhibited positive attitudes with respect to dealing with livestock that carries ticks in time to prevent family members from being bitten by ticks; cleaning up weeds and shrubs in the yard and/or around the house regularly; counselling related information about preventive strategies of SFTS in the lecture or propaganda, which were conducted in communities; participating in the free related detections of SFTS that were conducted by health departments. This may be explained by the fact that most respondents fear the disease as a result of living in small communities where cases and deaths have occurred. The majority of the participants exhibited positive to responses with regard to the prevention of infection by this disease. Due to the unavailability of a SFTS vaccine and increased public awareness is required regarding the risk factors of SFTS and possible preventive measures, which can aid the reduction of the exposure to the 
virus and the control of the spread of the disease. The successful application of preventive measures in livestock that carry ticks including the treatment of livestock with acaricides can decrease the risk of population exposure to ticks [28]. Cleaning up weeds and shrubs in the yard and/or around the house can eliminate tick habitats [28]. The participation in the free related detections of SFTS could be used by the local health authorities in the analysis of the epidemic condition and the development of preventive strategies. However, the relatively low proportion of respondents in the two phases with respect to adopting personal precautions when in contact with livestock indicated that the majority of the participants were unaware that domestic animals could act as amplifying hosts of SFTSV [29, 30].

More than $90 \%$ of respondents in the two phases believed that if someone was bitten by tick and developed symptoms of fever or other physical discomfort, they should raise vigilance and go to the hospital for treatment immediately. The strategy of seeking medical assistance rather than following detrimental self-treatment practices is notably important for the treatment of the disease in the absence of specific treatment. It would be difficult for physicians to diagnose SFTS due to the non-specific clinical symptoms [31]. Consequently, the early recognition and treatment of people who have been bitten by tick and experience any of the relevant disease symptoms is crucial for the decrease of the risk of serious complications. The majority $(68 \cdot 0 \%$ vs. $79 \cdot 4 \%)$ of the respondents in the two phases believed that someone should palsy tick with medical alcohol or iodine and then remove the tick slowly if he or she was bitten by a tick. By contrast, a small proportion of the respondents chose to squash the tick prior to removing it directly, which may force the tick's blood back into the skin. If the tick is infectious, people will become infected, as well. With regard to taking precautions when working outdoors, $56 \cdot 2 \%$ and $69 \cdot 9 \%$ of the respondents reported that they would wear longsleeved clothing and put cuffs into socks or shoes in order to avoid tick bite in the pre-intervention and post-intervention phases, respectively. Similarly, $31 \cdot 1 \%$ and $46 \cdot 3 \%$ of respondents reported that they would apply repellent in order to avoid tick bite in the two phases, respectively. The two personal precautionary measures were recommended by previous studies [7, 8]. The increased proportion of the effective practices that were used following the intervention programme might have been associated with the elevated level of knowledge. The previous study demonstrated that the level of knowledge was found to be the predictor of good practice [32]. At present, the decrease in the risk of the tick to human transmission is critical for outdoor workers in endemic areas. This decrease can be achieved by the application of long-sleeved clothing, the use of gloves and the minimal exposure of bare skin to tick-infested vegetation as well as the use of available insect repellents, including diethyltoluamide on bare skin.

The present study demonstrated that the intervention programme exhibited an optimal impact on knowledge, attitudes and practices of the population regarding SFTS in endemic areas. A similar study that was conducted in China indicated the effect of health education intervention on knowledge and practice with regard to dengue fever [33]. Similarly, the comprehensive intervention was reported to be an effective way to improve the health behaviors significantly and decrease the incidence rate of intestinal infection diseases effectively in the Guizhou Province [34]. Since the appropriate application of the intervention programme has been shown to improve KAP level regarding SFTS, the local health authorities and communities should strengthen and promote health education regularly.

\section{Study limitations and strengths}

This study provides the first evidence of the impact of an intervention programme on KAP concerning SFTS. However, this study has some limitations. First, some attendees were unresponsive and failed to complete the survey. Second, a lack of long-term follow-up to determine retention of knowledge gained. Third, although the assumed proportion of participant's knowledge on SFTS was in accordance with our results, it was based on the reference of other similar vectorborne disease [14] due to lack of awareness rate of SFTS reported, hence we also think it was a limitation of our study. Finally, we know that it would be better if the sample size in the post-intervention phase is matching with the sample size in the pre-intervention phase. Actually, due to the fact that most people work outdoors in April, it was hard to recruit the same number of participants in the post-intervention phase as in pre-intervention phase. Nevertheless, this study was successful in assessing baseline information regarding KAP related to SFTS prevention and in disseminating information regarding a locally important disease to many populations. 


\section{CONCLUSIONS}

A significant improvement in KAP of the population regarding SFTS was achieved in endemic areas of Lu'an city as a result of an intervention programme. Most of the respondents had an acceptable level of attitudes and practices about SFTS after an intervention programme was implemented, indicating the effect of our programme. However, the knowledge in relation to the most common symptoms and transmission modes of SFTS still needs to be improved. Based on previous results, we can conclude that administering an intervention programme can be considered as an effective tool for preventing infectious diseases as this method increases awareness of residents with regard to prevention and control of SFTS. Thus, we recommend that proactive health education via appropriated mass media should be strengthened and needs to be continuous. A set of local strategies should be formulated to promote the implementation of prevention and control measures aimed at enhancing the population's knowledge of SFTS, raising the self-protection awareness, minimising the exposure of SFTS and to promote positive attitudes and the best protective practices available. Project managers, health planners, policy makers and local governments need to apply greater effort to implementing SFTS control measures in communities to ensure those measures are proactive and effective. Further studies are required to confirm and extend the findings of the current study.

\section{ACKNOWLEDGEMENTS}

We would like to thank all the participants of the study, all the staff at Lu'an Municipality Center for Disease Control and Prevention who developed the intervention programme and the supporting team of Anhui Medical University who helped in data collection.

This project was supported by the Nature Science Foundation of the Anhui Provincial Higher Education Institutions of China (Grant No. KJ2017A187).

\section{DECLARATION OF INTEREST}

None.

\section{REFERENCES}

1. Yu XJ, et al. Fever with thrombocytopenia associated with a novel bunyavirus in China. The New England Journal of Medicine 2011; 364(16): 1523-1532.
2. Guo CT, et al. Epidemiological and clinical characteristics of severe fever with thrombocytopenia syndrome (SFTS) in China: an integrated data analysis. Epidemiology and Infection 2016; 144(6): 1345-1354.

3. Zhang $\mathbf{X}$, et al. An emerging hemorrhagic fever in China caused by a novel bunyavirus SFTSV. Science China Life Sciences 2013; 56(8): 697-700.

4. Kim KH, Ko MK. Seroprevalence of severe fever with thrombocytopenia syndrome in Southeastern Korea, 2015. Journal of Korea Medical Science 2017; 32(1): 29-32.

5. Kato H, et al. Epidemiological and clinical features of severe fever with thrombocytopenia syndrome in Japan, 2013-2014. PLoS ONE 2016; 11(10): e0165207.

6. Zhan J, et al. Current status of severe fever with thrombocytopenia syndrome in China. Virologica Sinica 2017; 32(1): 51-62.

7. Hu JL, et al. Risk factors for bunyavirus-associated severe fever with thrombocytopenia syndrome: a community-based case-control study. PLOS ONE 2016; 11(11): e0166611.

8. Ding F, et al. Risk factors for bunyavirus-associated severe fever with thrombocytopenia syndrome, China. PLoS Neglected Tropical Diseases 2014; 8(10): e3267.

9. Wang T, et al. Epidemiological characteristics and environmental risk factors of severe fever with thrombocytopenia syndrome in Hubei Province, China, from 2011 to 2016. Frontiers in Microbiology 2017; 8: 387.

10. Liang S, et al. Seroprevalence and risk factors for severe fever with thrombocytopenia syndrome virus infection in Jiangsu Province, China, 2011. The American Journal of Tropical Medicine and Hygiene 2014; 90(2): 256-259.

11. Li J, et al. A cross-sectional study of heat wave-related knowledge, attitude, and practice among the public in the Licheng District of Jinan City, China. International Journal of Environmental Research and Public Health 2016; 13(7): 648-663.

12. Sarkari B, Qasem A, Shafaf MR. Knowledge, attitude, and practices related to cutaneous leishmaniasis in an endemic focus of cutaneous leishmaniasis, Southern Iran. Asian Pacific Journal of Tropical Biomedicine 2014; 4(7): 566-569.

13. Abdi IH, et al. Knowledge, attitudes and practices (KAP) on rift valley fever among pastoralist communities of Ijara District, North Eastern Kenya. PLoS Neglected Tropical Diseases 2015; 9(11): e0004239.

14. Sayavong $\mathbf{C}$, et al. Knowledge, attitudes and preventive behaviors related to dengue vector breeding control measures among adults in communities of Vientiane, capital of the Lao PDR. Journal of Infection and Public Health 2015; 8(5): 466-473.

15. Khairy S, et al. Knowledge, attitude and practice about malaria in south-western Saudi Arabia: a householdbased cross-sectional survey. Journal of Infection and Public Health 2017; 10(5): 499-506.

16. Tuohetamu S, et al. The knowledge, attitudes and practices on influenza among medical college students in Northwest China. Human Vaccines \& Immunotherapeutics 2017; 13(7): 1688-1692.

17. Gu H, Jiang Z. Knowledge, attitudes, and practices regarding avian influenza A (H7N9) among mobile 
phone users: a survey in Zhejiang Province, China. JMIR mHealth uHealth 2015; 3(1): e15.

18. Chan EY, et al. Knowledge, attitudes, and practices of Hong Kong population towards human A/H7N9 influenza pandemic preparedness, China, 2014. BMC Public Health 2015; 15: 943.

19. Valdivieso F, et al. Knowledge, attitudes, and practices regarding hantavirus disease and acceptance of a vaccine trial in rural communities of southern Chile. Human Vaccines \& Immunotherapeutics 2017; 13(4): 808-815.

20. Mouchtouri VA, et al. Knowledge, attitudes, and practices about the prevention of mosquito bites and zika virus disease in pregnant women in Greece. International Journal of Environmental Research and Public Health 2017; 14(4): 367-375.

21. Lyu Y, et al. Seroprevalence and risk factors of severe fever with thrombocytopenia syndrome virus infection in endemic areas. Infectious Diseases (London, England) 2016; 48(7): 544-549.

22. Fisher JD, et al. Changing AIDS risk behavior: effects of an intervention emphasizing AIDS risk reduction information, motivation, and behavioral skills in a college student population. Health Psychology 1996; 15 (2): 114-123.

23. Fisher JD, et al. Information-motivation-behavioral skills model-based HIV risk behavior change intervention for inner-city high school youth. Health Psychology 2002; 21(2): 177-186.

24. Bian C, et al. A study on the application of the information-motivation-behavioral skills (IMB) model on rational drug use behavior among second-level hospital outpatients in Anhui, China. PLOS ONE 2015; 10(8): e0135782.

25. Al-Dubai SA, et al. Factors affecting dengue fever knowledge, attitudes and practices among selected urban, semi-urban and rural communities in Malaysia. The Southeast Asian Journal of Tropical Medicine and Public Health 2013; 44(1): 37-49.

26. Wong LP, et al. Factors affecting dengue prevention practices: nationwide survey of the Malaysian public. PLOS ONE 2015; 10(4): e0122890.

27. Lugova H, Wallis S. Cross-sectional survey on the dengue knowledge, attitudes and preventive practices among students and staff of a public university in Malaysia. Journal of Community Health 2017; 42(2): 413-420.

28. Aslam S, et al. Crimean-Congo hemorrhagic fever: risk factors and control measures for the infection abatement. Biomedical Reports 2016; 4(1): 15-20.

29. Liu Q, et al. Severe fever with thrombocytopenia syndrome, an emerging tick-borne zoonosis. The Lancet Infectious Diseases 2014; 14(8): 763-772.

30. Liu L, et al. [Epidemiologic analysis on severe fever with thrombocytopenia syndrome in Hubei province, 2010]. Zhonghua liu xing bing xue za zhi = Zhonghua liuxingbingxue zazhi 2012; 33(2): 168-172.

31. Sun Y, et al. Early diagnosis of novel SFTS bunyavirus infection by quantitative real-time RT-PCR assay. Journal of clinical Virology 2012; 53(1): 48-53.

32. Castro M, et al. The relationship between economic status, knowledge on dengue, risk perceptions and practices. PLoS ONE 2013; 8(12): e81875.

33. Jiang J, Xu Y, Yang XB, Zhang H. The effect evaluation of health education intervention of dengue fever prevention and control in Yichang City, Hubei Province. Chinese Journal of Health Education 2012; 28(1): 43-45 (in Chinese).

34. Ling M, et al. Effect evaluation on comprehensive intervention on intestinal infection diseases in Guizhou Province. Chinese Journal of Health Education 2012; 28(9): 714-718 (in Chinese). 